\title{
Right Ventricular Mechanical Changes after Septal Myectomy for Obstructive Hypertrophic Cardiomyopathy: Vector Velocity Imaging Study
}

\author{
Hala Mahfouz Badran',2, Ghada Soltan1, Naglaa Faheem¹,2, Mohamed Ezzat Enait ${ }^{1}$, \\ Magdi H. Yacoub ${ }^{2,3}$ \\ ${ }^{1}$ Cardiology Department Menoufia University, Al Minufya, Egypt \\ ${ }^{2}$ The BAHCM National Program, Aswan Heart Center, Egypt \\ ${ }^{3}$ Imperial College, London, UK \\ Email: ebnezat@gmail.com
}

How to cite this paper: Badran, H.M., Soltan, G., Faheem, N., Enait, M.E. and Yacoub, M.H. (2019) Right Ventricular Mechanical Changes after Septal Myectomy for Obstructive Hypertrophic Cardiomyopathy: Vector Velocity Imaging Study. World Journal of Cardiovascular Diseases, 9, 467-488.

https://doi.org/10.4236/wjcd.2019.97042

Received: March 14, 2019

Accepted: July 28, 2019

Published: July 31, 2019

Copyright $\odot 2019$ by author(s) and Scientific Research Publishing Inc. This work is licensed under the Creative Commons Attribution International License (CC BY 4.0).

http://creativecommons.org/licenses/by/4.0/

\begin{abstract}
Objectives: The impairment of right ventricular (RV) myocardial mechanics is evident in hypertrophic cardiomyopathy (HCM). It is independently influenced by LV mechanics and correlated to the severity of LV phenotype. We investigated the changes in RV global and regional deformation following surgical septal myectomy using vector velocity imaging (VVI). Methods: 25 HCM patients, $68 \%$ males with mean age (34.5 \pm 12 years) were examined before and within two months after surgical myectomy using VVI. In addition to conventional echocardiographic parameters, peak systolic strain $\left(\varepsilon_{s y s}\right)$, strain rate (SR) and time to peak $\varepsilon_{\text {sVs }}$ (TTP) of regional RV free wall (RVFW) \& septal walls were analyzed in longitudinal (long) directions from apical four-chamber view and their $(\Delta)$ changes were calculated. Similar parameters were quantified in LV from apical $2 \& 4 \mathrm{CH}$ views. Intra-V-delay was defined as SD of TTP and inter-V dyssynchrony was estimated from TTP difference between the most delayed LV segment \& RVFW. Results: All study patients showed improvement of their functional class from NYHA class III to class I and reduction of LVOT gradient to below $20 \mathrm{mmHg}$ except one patient who had $30 \mathrm{mmHg}$ gradients at rest. There was significant reduction of septal thickness, left atrial diameter \& volume, LVOT gradient, LVMI, severity of mitral regurgitation, tricuspid annular velocities $(\mathrm{P}<0.0001)$, RV diameter $(\mathrm{P}$ $<0.02)$ and increase in LV internal dimensions $(\mathrm{P}<0.001)$ post myectomy. However, there was significant reduction of RV and LV systolic mechanics; RV global $\varepsilon_{s y s} \%$ (from $-16.1 \% \pm 4.4 \%$ to $-12.9 \% \pm 2.9 \%, \mathrm{P}<0.0001$ ) and LV global $\varepsilon_{s y s} \%$ : from $-11.6 \% \pm 2.8 \%$ to $\left.-9.4 \% \pm 2.2 \%, \mathrm{P}<0.0001\right)$ respectively. The magnitude of reduction of RV strain $\left(\Delta \mathrm{RV} \varepsilon_{\text {sys }} \%, \Delta \mathrm{SR}_{\text {sys }}\right)$ was directly
\end{abstract}


correlated LV maximal wall thickness $(\mathrm{r}=0.46, \mathrm{P}<0.01)$ and $\Delta \mathrm{RV}$ dyssynchrony (TTP-SD) $(\mathrm{r}=0.4, \mathrm{P}<0.05)$ and negatively correlated to age $(\mathrm{r}=$ $-0.46, \mathrm{P}<0.02)$, pre-op RV SR $\mathrm{sys}_{\text {s }}(\mathrm{r}=-0.52, \mathrm{P}<0.01)$ and pre-op LV EF $\%(\mathrm{r}$ $=-0.43, \mathrm{P}<0.03)$. Meanwhile, the reduction in RV diastolic mechanics: $\Delta \mathrm{RV}$ $\mathrm{SR}_{\mathrm{e}} \& \mathrm{SR}_{\mathrm{a}}$ were directly correlated to PAP and LVOT gradient before surgery $(r=0.62, P<0.002)$. Conclusion: Despite the improvement of patient functional status and reduction LVOT gradient, RV mechanics shows further deterioration after surgical myectomy. The magnitude of reduction is modestly related to cardiac phenotype and pre-op mechanical function.

\section{Keywords}

Right Ventricle, Deformation Imaging, Hypertrophic Cardiomyopathy

\section{Introduction}

Hypertrophic cardiomyopathy (HCM), the most common genetically inherited heart muscle disease, affects the right ventricle (RV) because of the anatomically hypertrophied septum and conceivably by extension of the myopathic process to the RV [1]. Magnetic resonance studies by Maron et al. [2] demonstrated that the RV wall thickness was increased in patients with HCM compared to controls. In a third of patients with HCM, RV wall thickness and/or mass were increased, including about $10 \%$ with extreme RV wall hypertrophy ( $>10 \mathrm{~mm}$ ). Most patients with HCM (53\%) had diffuse RV hypertrophy involving all three segments of the RV.

In hypertrophic obstructive cardiomyopathy (HOCM), invasive interventions are usually considered when pharmacotherapy either fails to control symptoms or is not tolerated [3] [4].

Recent studies have shown that cardiac dysfunction is not limited to the left ventricle, impairment of RV mechanics is evident in HCM using feature tracking. It is independently influenced by LV deformation and correlated to the severity of LV phenotype [3].

With the development of new ultrasound-based technologies to assess LV mechanics, the assessment of cardiac performance has been revolutionized. Recent speckle-tracking-based velocity vector imaging (VVI) enables the evaluation of regional or global myocardial deformation by measuring the strain, and strain rate (SR). VVI integrates frame-to-frame changes with the geometric shift of each speckle and thus allows the simultaneous evaluation of multiple components of myocardial deformation [5]. It has strikingly advanced our understanding of the pathophysiology of HCM, and added new dimension in quantifying myocardial remodeling that might occur after surgical myectomy. It improved substantively our understanding of ventricular mechanics [6].

Data on RV functional changes in HCM patients following myectomy are limited [7] [8]. More specifically, to our knowledge, no comparative study was 
characterizing the changes in RV strain pattern after surgical myectomy. Accordingly, in this cross-sectional study, we sought to: a) characterize regional and global RV strain in a cohort of patients with HCM before and after surgical myectomy using vector velocity imaging; b) investigate the relationship of RV strain patterns post myectomy to LV deformation and cardiac phenotype in this population.

\section{Patients and Methods}

\subsection{Study Population}

Twenty-five patient undergoing septal myectomy without concomitant surgery (valve repair or replacement or maze procedure) done at Aswan Hear Center (Aswan, Egypt) for symptomatic HOCM were studied. They were examined in a single centre (Yacoub Research Unite, Menoufia University, Egypt and as a part of the BA-HCM National Program). Patients were subjected to full baseline clinical records; echocardiograms obtained two months before myectomy and on follow-up 3 to 6 months after myectomy, and echocardiographic studies adequate for strain analysis in both baseline and follow-up studies.

The control group consisted of 33 healthy subjects without family history of hypertrophic cardiomyopathy (HCM) and normal echocardiographic findings with similar gender and age distributions. Patients were enrolled in the study after their informed consent

We excluded patients who had 1) poor echo window; 2) clinical or echocardiographic evidence of other cardiac diseases such as CAD or congenital heart disease and 3) NYHA functional class IV.

The study was approved by the Ethics Committee of Menoufia University Hospitals was obtained. The diagnosis of HCM was based on conventional echocardiographic demonstration of a non-dilated, hypertrophic LV ( $\geq 15 \mathrm{~mm})$ in the absence of other cardiac or systemic diseases capable of producing the magnitude of hypertrophy evident [2] [3] [7]. Myectomy was offered to patients with unacceptable symptoms despite maximally tolerated pharmacotherapy with LVOT gradients $\geq 50 \mathrm{~mm} \mathrm{Hg}$ at rest or after provocation (Valsalva maneuver, inhalation of amyl nitrate, or ventricular premature beat), as measured during Doppler echocardiography or cardiac catheterization [3] [7]. Patients were selected for surgical septal myectomy rather than alcohol septal ablation on the basis of their ages, comorbidities, and preferences [8].

\subsection{Clinical Evaluation}

Traditional clinical variables of interest included demographics (age and sex), functional assessment (New York Heart Association [NYHA] class), pertinent history (family history of sudden cardiac death, history of arrhythmias, and history of unexplained syncope, Patient charts were retrospectively reviewed for clinical and demographic variables. All subjects underwent 12-lead electrocardiography, standard echocardiography (M-mode, 2-DEcho, Doppler), 2D strain imaging using VVI for measurement of RV and LV longitudinal strain (LS). 


\subsection{Conventional Echocardiography}

Echocardiographic examinations were performed with all subjects positioned in the left lateral decubitus, by the same operator (HB) in the parasternal long, short-axis, apical 2- \& 4-chamber views using standard transducer positions. Esaote Mylab Gold 30 ultrasound system (Esaote S.p.A, Florence, Italy) equipped with a multi-frequency 2.5 - 3.5 $\mathrm{MHz}$ phased-array transducer was utilized [9] [10]. RV end diastolic diameter and wall thickness, LV end diastolic (LVEDD), end systolic diameter (LVESD), septum (SPT), posterior wall thickness (PWT), ejection fraction (EF \%) and left atrial (LA) diameter \& volume and volume index were all measured in accordance with the recommendations of the American Society of Echocardiography [11]. The magnitude and extent of hypertrophy in both ventricles were assessed in all views.

Color flow mapping and continuous-wave Doppler was used to define resting LVOT, mid cavity and to estimate pulmonary artery pressure (PAP) from tricuspid regurgitation velocity (Bernoulli equation).

Additionally, the diastolic function was assessed using the standard indexes of transmitral and transtricuspid flow and TDI. The TDI program was set in pulsed-wave Doppler mode. Motion of mitral annulus was recorded in the apical four-chamber view at a frame rate of 80 to 140 frames per second [11]. Peak early (E) and late (A) transmitral (Em \& Am) and trantricuspid (Et \& At) filling velocities were measured from mitral and tricuspid inflow velocities. Peak systolic $\left(S_{a}\right)$, early diastolic $\left(E_{a}\right)$ and atrial diastolic $\left(A_{a}\right)$ annular velocity were obtained using tissue Doppler imaging (TDI) by placing a myocardial TDI sample volume at the RVFW and lateral mitral annulus in the apical 4-chamber view. All velocities were recorded for three consecutive cardiac cycles during end-expiratory phase, and the results were averaged. All TDI signals were recorded at horizontal time sweep set at $50-100 \mathrm{~mm} / \mathrm{s}$ accordingly to current guidelines [12]. The $\mathrm{E}_{\mathrm{m}} / \mathrm{E}_{\mathrm{am}}$ and $\mathrm{E}_{\mathrm{t}} / \mathrm{E}_{\mathrm{at}}$ ratio were calculated. This ratio has been reported to correlate with LV and RV filling pressure [12] [13].

\subsection{Analysis of RV and LV Deformation}

LV longitudinal strain analysis was included as part of the routine TTE evaluation. Strain measurement was based on the VVI: the global myocardial deformation was evaluated from standard $2 \mathrm{D}$-images at frame rate $(70 \pm 20 \mathrm{~F} / \mathrm{s})$ at rest and adjusted depending on the heart rate to $80 \pm 23 \mathrm{~F} / \mathrm{s}$. The images were stored, during three cardiac cycles, in digital format for subsequent offline analysis. To avoid excessive translational motion seen the clips always captured with complete breath-holding during expiration. Tracking and subsequent strain calculations were performed with the software package Esaote-X-Strain based on previously validated algorithm [14] [15]. Scanning was performed from the apex to acquire best apical $2 \& 3$ and 4 chamber views.

Border tracking of the left ventricle (LV) and right ventricle (RV) was manually traced in the recorded clips of apical $4 \mathrm{CH} \& 2 \mathrm{CH}$ views, with good quality 
ECG signal and a frame rate between 40 - $70 \mathrm{fps}$. They were then stored for offline analysis using X-Straine software. VVI is dedicated software that derives longitudinal myocardial velocity, strain $(\varepsilon)$, strain rate (SR) from digitized $2 \mathrm{D}$ video clips. The endocardial border is automatically drawn at end-diastole using a point-and-click approach [6] [9] [12].

Right ventricular longitudinal strain data were prospectively extracted in a blinded manner from images acquired in the four-chamber view. A point and click approach was utilized to identify $3 \mathrm{RV}$ anchor points (both annuli and RV apex) enabling the software to track the endocardial contour automatically. Subsequently, tracking was visually inspected throughout systole to ensure adequate border detection and the endocardial contours adjusted manually as necessary to further optimize tracking.

Reproducibility for quantitative RV indices has been validated [12] [13].

Longitudinal strains $\left(\varepsilon_{\text {sys }}\right)$ and strain rate $(\mathrm{SR})$ during systole $\left(\mathrm{SR}_{\text {sys }}\right)$, early $\left(\mathrm{SR}_{\mathrm{e}}\right)$ and late diastole $\left(\mathrm{SR}_{\mathrm{a}}\right)$ for each individual segment were measured and averaged to obtain RV septal (basal mid and apical walls) and lateral RV free wall (basal, mid and apical) values. RV global longitudinal strain (GLS) was estimated by averaging septal and lateral right ventricular segmental strains.

Similarly, for LV it was tracked from the septal side of the mitral annulus to lateral side in apical $4 \mathrm{CH}$ and from anterior to the inferior side in the apical $2 \mathrm{CH}$. Velocity, strain, and SR graphics were automatically obtained [10].

To estimate LV mechanical dyssynchrony, time to peak strain (TTP) was measured from regional longitudinal strain curves for each ventricular segment, as time from the beginning of Q wave of ECG to the time to peak $\varepsilon_{s y s}$ Electromechanical delay (TTP-d) was measured as the difference of time to peak systolic strain in $12 \mathrm{LV}$ myocardial segments and $6 \mathrm{RV}$ myocardial segments (difference between the longest and shortest TTP) [9] [10] [11]. Mechanical dyssynchrony was defined as the standard deviation of the averaged time-to-peak-strain (TTP-SD) [9] [10] [11].

\subsection{Surgical Technique}

All patients underwent myectomy by the standard described procedure [12]. The clinical status of patients was monitored during the follow-up visits and echocardiographic examination and strain analysis were performed within 2 months after surgical myectomy.

\subsection{Statistical Analysis}

Data were presented as numbers (\%) or mean \pm SD. Categorical variables were presented as percentages and compared with two-tailed Chi-square or Fisher's exact test, as appropriate. Continuous variables were presented as the mean $\pm \mathrm{SD}$ and compared with unpaired Student's t-test. Quantitative variables were correlated by the use of Pearson's correlation coefficient " $r$ ". All tests were 2-tailed and $\mathrm{p}$-value $<0.05$ was considered statistically significant. Statistical analysis was performed using commercially available statistical software SPSS for MAC version 23. 


\section{Results}

\subsection{Patients Characteristics}

Surgical Myectomy was successfully done for all twenty-five (25) patients. Twenty patients (80\%) developed LBBB and one patient (4\%) developed RBBB post operatively. It had been carried out at Magdi Yacoub Aswan Heart center, in the period from August 2014 to October 2018.

Clinical characteristics of the study population were depicted in Table 1 . The age was ranged from 15 to 62 and mean was $34.4 \pm 12.6$ years, and $72 \%$ were men. All patients were treated with B blockers and $(60 \%)$ were treated using a combination of b-blockers and/or calcium channel blocker, all patients had New York Heart Association class III symptoms. Major symptoms included dyspnea (96\%), chest pain (12\%), and syncope (4\%). All patients had basal septal myectomy, with no other concomitant surgery was performed. There was no intraoperative or early mortality (within 30 days postsurgery). All patients had prominent basal septal hypertrophy.

Post-myectomy complete atrioventricular block necessitating permanent pacing occurred in $4 \%$ of patients, and new complete left bundle branch block occurred in 21 patients (84\%).

Table 1. Patients clinical characterstics.

\begin{tabular}{|c|c|c|c|}
\hline Variable & All HCM $(n=313)$ & Variable & All HCM $(n=313)$ \\
\hline \multicolumn{4}{|l|}{ Age } \\
\hline$<40 \mathrm{ys}$ & $17(68 \%)$ & Duration of illness (ms) & $15.5 \pm 12.6$ \\
\hline$\geq 40$ ys & $8(32 \%)$ & & \\
\hline Gender & & Family history $(+)$ & $6(24 \%)$ \\
\hline Male & $16(64 \%)$ & & \\
\hline Female & $9(36 \%)$ & & \\
\hline $\mathrm{BSA} \mathrm{m}^{2}$ & $1.89 \pm 0.17$ & Duration of follow up & $3.24 \pm 1.21$ \\
\hline Medications & & Symptoms: & \\
\hline B Blockers & $25(100 \%)$ & Dyspnea & $24(96 \%)$ \\
\hline B-blockers & $15(60 \%)$ & Fatigue & $4(16 \%)$ \\
\hline +Ca blockers & & Chest pain & $3(12 \%)$ \\
\hline Functional class & $25(100 \%)$ & Palpitation & $2(8 \%)$ \\
\hline NYHA III & & Syncope & $1(4 \%)$ \\
\hline Index & $24(96 \%)$ & $\mathrm{HR}$ & $72 \pm 12$ \\
\hline SBP & $110 \pm 25$ & DBP & $73 \pm 4$ \\
\hline LVH: & & MR severity & \\
\hline Asymmetric & $23(92 \%)$ & I & $5(20 \%)$ \\
\hline Concentric & $1(4 \%)$ & II & $13(52 \%)$ \\
\hline \multirow[t]{2}{*}{ Localized septal } & $1(4 \%)$ & III & $5(20 \%)$ \\
\hline & & IV & $2(8 \%)$ \\
\hline
\end{tabular}


After myectomy, all patients improved their functional capacity by at least one class.

\subsection{Conventional Echocardiography}

Baseline and post myectomy conventional echocardiographic parameters are shown in Table 2. At baseline, patients demonstrated asymmetric septal hypertrophy, varying levels of LVOT obstruction (ranged from 115 to $56 \mathrm{mmHg}$ ), mild to moderate MR in $80 \%$ of patients, and normal LV systolic function. Left atrial volume index was significantly increased.

Post myectomy there was dramatic decrease in the LVOT gradient to nonobstructive levels $(11.4 \pm 5.6$ vs $67.9 \pm 14.5 ; \mathrm{p}<0.0001)$. There was significant decrease in LA diameter, LA volume and its volume index (LAVI) post myectomy when compared with per myectomy (39.8 \pm 5.6 vs $46.4 \pm 7.9$; p 0.000), (62.2 \pm 19.9 vs $86.5 \pm 33.3 ; \mathrm{p}<0.000)$, and $(32.8 \pm 9.2$ vs $46.7 \pm 20.9 ; \mathrm{p}<0.000)$ respectively.

Similarly LVESD increased significantly post myectomy $(27.1 \pm 4.1$ vs $22.0 \pm$ 4.1; $\mathrm{p}<0.000)$ while LVEDD the increase did not reach statistical significance $(44.0 \pm 6.7$ vs $41.2 \pm 5.9 ; \mathrm{p}<0.060)$. Additionally IVS, and LVPW were significantly reduced post myectomy $19.1 \pm 4$ vs $26.1 \pm 5 ; \mathrm{p}<0.000)$ and $(10.5 \pm 2.1$ vs $12 \pm 3.8 ; \mathrm{p}<0.050)$ respectively. Consequently, there was significant decrease in LV mass (LVM) and LV mass index (LVMI) post myectomy when compared with per myectomy $(318.6 \pm 107.9$ vs $449.5 \pm 128.1 ; \mathrm{p}<0.000)$ and $(165 \pm 51.3$ vs $240.4 \pm 62.3 ; \mathrm{p}<0.000)$ respectively

LV ejection fraction modestly but significantly decreased ( $68.3 \pm 5.4$ vs $75.8 \pm$ 8.2; $\mathrm{p}<0.0001)$, however, it is remaining within the normal range. Associated changes included a decrease in the grade of mitral regurgitation and a reduction in the left atrial volume index. Diastolic mitral inflow parameters were similar to premyectomy values. Tissue Doppler velocities demonstrated an increase in mitral E' and a similar decrease in the E/E' ratio (Table 2, Figure 1).

Table 2. Comparison of clinical and conventional echocardiographic parameters before and after myectomy.

\begin{tabular}{cccc}
\hline & Pre-myectomy & Post-myectomy & P Value \\
\hline HR $(\mathrm{b} / \mathrm{m})$ & $69.4 \pm 9$ & $65.8 \pm 10$ & 0.19 \\
SBP $(\mathrm{mmHg})$ & $115 \pm 9$ & $112 \pm 6$ & 0.11 \\
DBP (mmHg) & $72 \pm 5$ & $71 \pm 4$ & 0.42 \\
Symptoms & & & \\
Dyspnea & $23(92 \%)$ & 0 & $<0.0001$ \\
Syncope & $3(12 \%)$ & 0 & \\
Chest pain & $2(8 \%)$ & 0 & \\
Dizziness & $1(4 \%)$ & 0 & \\
\hline
\end{tabular}




\section{Continued}

\begin{tabular}{|c|c|c|c|}
\hline \multicolumn{4}{|l|}{ NYHA class } \\
\hline I & $0.0 \%$ & $25(100 \%)$ & \multirow{2}{*}{$<0.0001$} \\
\hline II & 0 & $0.0 \%$ & \\
\hline III-IV & $25(100 \%)$ & $0.0 \%$ & \\
\hline $\mathrm{LAD}(\mathrm{mm})$ & $46.4 \pm 8$ & $39.8 \pm 5.6$ & 0.000 \\
\hline $\mathrm{LAV}(\mathrm{ml})$ & $86.5 \pm 33$ & $62.2 \pm 20$ & 0.000 \\
\hline $\operatorname{LAVI}\left(\mathrm{ml} / \mathrm{m}^{2}\right)$ & $46.7 \pm 21$ & $32.8 \pm 9.2$ & 0.000 \\
\hline LV ESD $(\mathrm{mm})$ & $22.0 \pm 4.1$ & $27.1 \pm 4.1$ & 0.000 \\
\hline LV EDD (mm) & $41.2 \pm 6$ & $44.0 \pm 6.7$ & 0.060 \\
\hline FS (\%) & $47.5 \pm 8$ & $38.2 \pm 5$ & 0.000 \\
\hline LV EF (\%) & $75.8 \pm 8.2$ & $68.3 \pm 5.4$ & 0.001 \\
\hline IVS (mm) & $26.1 \pm 5$ & $19.1 \pm 4.1$ & 0.000 \\
\hline LVPW (mm) & $12 \pm 3.8$ & $10.5 \pm 2.1$ & 0.051 \\
\hline $\operatorname{LVM}(\mathrm{g})$ & $449 \pm 128$ & $318 \pm 107$ & 0.000 \\
\hline LVMI $\left(\mathrm{gm} / \mathrm{m}^{2}\right)$ & $240 \pm 62$ & $169 \pm 51$ & 0.000 \\
\hline LVOTG (mmHg) & $68 \pm 14.5$ & $11.4 \pm 5.6$ & 0.000 \\
\hline RV diameter (mm) & $22.1 \pm 3.3$ & $20.9 \pm 3.5$ & 0.029 \\
\hline Grade of MR & $2.2 \pm 0.9$ & $0.6 \pm 0.6$ & 0.000 \\
\hline Mitral E (cm/s) & $71.2 \pm 32.6$ & $66.6 \pm 26.8$ & 0.355 \\
\hline Mitral A $(\mathrm{cm} / \mathrm{s})$ & $66 \pm 17.2$ & $66.8 \pm 21.2$ & 0.841 \\
\hline $\mathrm{DT}(\mathrm{ms})$ & $209 \pm 49.5$ & $208.1 \pm 33.3$ & 0.869 \\
\hline Grade of TR & $1.7 \pm 0.7$ & $1.0 \pm 0.5$ & 0.324 \\
\hline Mitral E/Ema & $13.5 \pm 9.1$ & $9.4 \pm 2.4$ & 0.027 \\
\hline PAP (mmHg) & $28.4 \pm 8.2$ & $24.4 \pm 5.6$ & 0.055 \\
\hline $\operatorname{Sma}(\mathrm{cm} / \mathrm{s})$ & $8.7 \pm 1.7$ & $7.9 \pm 2.8$ & 0.174 \\
\hline $\operatorname{Ema}(\mathrm{cm} / \mathrm{s})$ & $6 \pm 2$ & $7.3 \pm 2.6$ & 0.043 \\
\hline Ama $(\mathrm{cm} / \mathrm{s})$ & $9.5 \pm 2.5$ & $9 \pm 2.2$ & 0.445 \\
\hline Sta $(\mathrm{cm} / \mathrm{s})$ & $12.6 \pm 2.6$ & $8.7 \pm 3.6$ & 0.000 \\
\hline $\operatorname{Eta}(\mathrm{cm} / \mathrm{s})$ & $11.6 \pm 4.8$ & $6.8 \pm 2.4$ & 0.000 \\
\hline Ata $(\mathrm{cm} / \mathrm{s})$ & $14.8 \pm 5.2$ & $10.3 \pm 2.9$ & 0.002 \\
\hline
\end{tabular}

HR: heart rate; SBP: systolic blood pressure, DBP: diastolic blood pressure. LA: left atrium, LV: left ventricle, ESD: end systolic diameter, EDD: end diastolic diameter, FS: fractional shortening, EF: Ejection fraction, IVSd: inter-ventricular septal diameter, PWd: diastolic posterior wall diameter, LVM: left ventricular mass, LVMI: left ventricular mass index, LVOT PG: left ventricular outflow tract pressure gradient, RV: right ventricle, MR: mitral regurgitation, E: early mitral inflow diastolic velocity, A: late diastolic mitral inflow velocity, DT: deceleration time, PAP: estimated pulmonary artery pressure, $\mathrm{S}_{\mathrm{ma}}$ : peak systolic velocity of mitral annulus; $\mathrm{E}_{\mathrm{ma}}$ : early diastolic velocity of mitral annulus; $\mathrm{A}_{\mathrm{ma}}$ : atrial diastolic velocity of mitral annulus; $S_{t a}$ : peak systolic velocity of tricuspid annulus; $E_{t a}$ : early diastolic velocity of tricuspid annulus; $A_{t a}$ : atrial diastolic velocity of tricuspid annulus. 


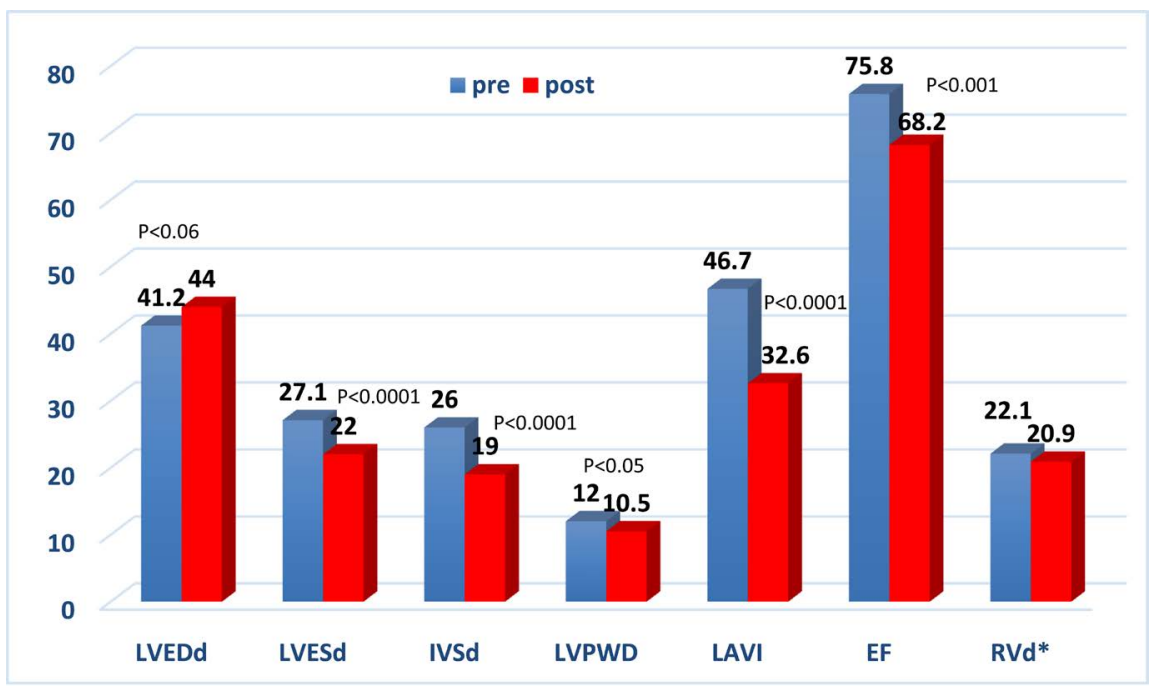

LVEDD: left ventricle end diastolic diameter, LVESD: left ventricle end systolic diameter, IVSD: diastolic inter-ventricular septal diameter, PWD: diastolic posterior wall diameter, FS: fractional shortening, EF: Ejection fraction; RVd: right ventricular diameter.

Figure 1. Comparison between LV dimensions and function pre- and post-myectomy.

\subsection{Tissue Doppler Velocities of Mitral and Tricuspid Valve Annuli}

Diastolic mitral inflow parameters were similar to premyectomy values. However, tissue Doppler velocities demonstrated a significant increase in mitral annular $\mathrm{E}_{\mathrm{m}}$ post-myectomy when compared with pre-myectomy (7.3 \pm 2.6 vs $6.0 \pm 2$; $\mathrm{p}<0.043)$. This is associated with significant decrease in $\mathrm{E} / \mathrm{E}_{\mathrm{m}}$ ratio $(\mathrm{P}<0.03)$. Similarly, $\mathrm{S}_{\mathrm{t}}, \mathrm{E}_{\mathrm{t}}$ and $\mathrm{A}_{\mathrm{t}}$ of tricuspid annular velocity were significantly increased post myectomy $(8.7 \pm 3.6$ vs $12.6 \pm 2.6 ; \mathrm{p}<0.000),(6.8 \pm 2.4$ vs $11.6 \pm 5 ; \mathrm{p}<$ $0.001)$ and $(10.3 \pm 3$ vs $14.8 \pm 5.2 ; \mathrm{p}<0.002)$ respectively (Figure 2$)$.

\subsection{Right Ventricular Mechanics}

RV segmental and GLS ( $\left.\varepsilon_{s y s} \%\right)$ pre and post-surgical myectomy of the study population are summarized in Table 3. Pre-procedural RV mechanics: septal strain was significantly lower compared with RVFW segments and particularly demonstrated a high ( $-27 \%$ to $11 \%)$ basal-to-apical gradient.

Post myectomy, there was significant reduction in RV septum strain $(-10.7 \pm$ 3.3 vs $-12.3 \pm 3.8 ; \mathrm{p}<0.05)$, RVFW basal segment $(-20.6 \pm 8.2$ vs $-27.3 \pm 13 ; \mathrm{p}$ $0.008)$, mid $(-15.5 \pm 5.3$ vs $-20.4 \pm 10.3 ; \mathrm{p}<0.01)$ segments, apical segment $(-15.7 \pm 4.7$ vs $-19.9 \pm 8.2 ; \mathrm{p}<0.007)$ and global RV $\mathcal{\varepsilon}_{\text {sys }} \%(-12.9 \pm 2.9$ vs -16.1 $\pm 4.7 ; \mathrm{p}<0.000$ ) when compared with pre-myectomy. No significant change in $\mathrm{RV}$ electromechanical delay (TTP-d) or RV intra-ventricular dyssynchrony following surgical myectomy (Table 3, Figures 3-6).

\subsection{RV Strain Rate Parameters}

Table 4 is demonstrating changes in $\mathrm{RV}$ peak systolic strain rate $\left(\mathrm{SR}_{\mathrm{sys}}\right)$, early $\left(\mathrm{SR}_{\mathrm{e}}\right)$ and late $\left(\mathrm{SR}_{\mathrm{a}}\right)$ diastolic strain rate following myectomy. Post operative, 


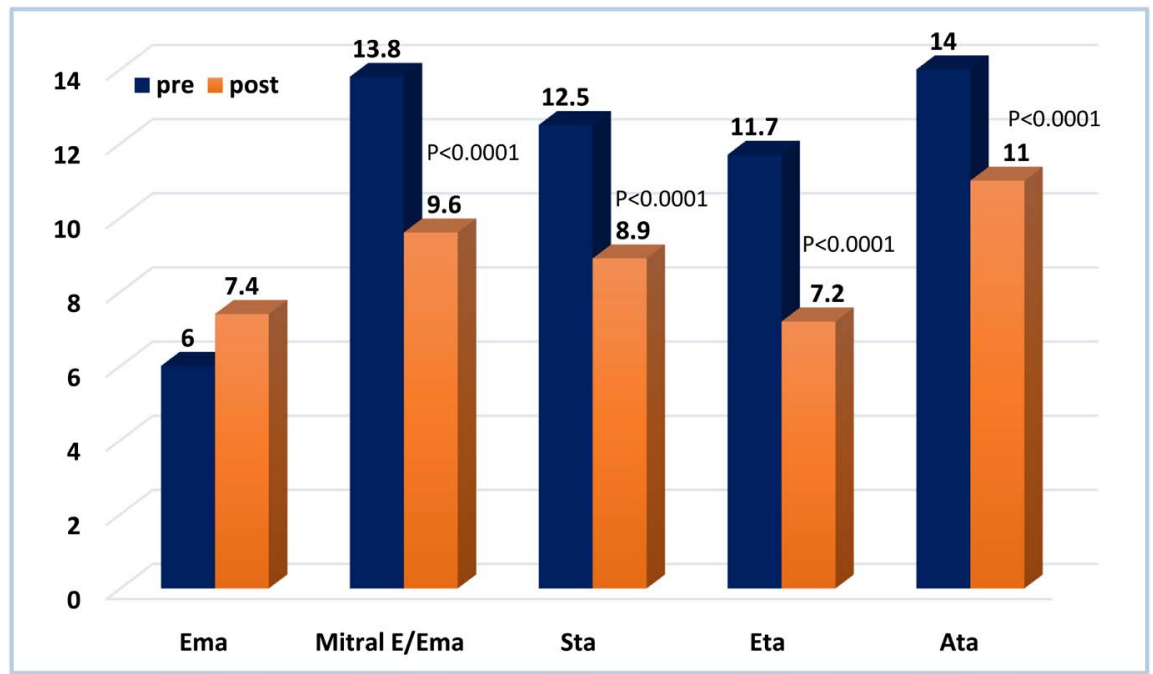

Ema: early diastolic velocity of mitral annulus; E: early diastolic mitral inflow velocity; Sta: peak systolic velocity of tricuspid annulus; Eta: early diastolic velocity of tricuspid annulus; Ata: atrial diastolic velocity of tricuspid annulus.

Figure 2. Comparison between tissue Doppler velocities of mitral and tricuspid annuli pre- and post-surgical myectomy.

Table 3. Comparison between RV longitudinal systolic strain and mechanical dyssynchrony pre- and post-myectomy.

\begin{tabular}{|c|c|c|c|}
\hline & Pre-myecyomy & Post-myecyomy & P Value \\
\hline RV Basal sept $\varepsilon_{s y s}(\%)$ & $-13.2 \pm 5.1$ & $-11.3 \pm 4.9$ & 0.145 \\
\hline RV Mid sept $\varepsilon_{s y s}(\%)$ & $-12.7 \pm 4.3$ & $-11.0 \pm 3.8$ & 0.084 \\
\hline RV Apical sept $\varepsilon_{s y s}(\%)$ & $-11.1 \pm 4$ & $-9.6 \pm 2.8$ & 0.128 \\
\hline Mean sept $\varepsilon_{s y s}(\%)$ & $-12.3 \pm 3.8$ & $-10.7 \pm 3.3$ & 0.050 \\
\hline RV Basal RVFW $\varepsilon_{s y s}(\%)$ & $-27.3 \pm 13$ & $-20.6 \pm 8.2$ & 0.008 \\
\hline RV Mid RVFW $\varepsilon_{s y s}(\%)$ & $-20.4 \pm 10.3$ & $-15.5 \pm 5.3$ & 0.018 \\
\hline RV Apical RVFW $\varepsilon_{s y s}(\%)$ & $-11.9 \pm 5.3$ & $-9.6 \pm 3.3$ & 0.858 \\
\hline Mean RVFW $\varepsilon_{s y s}(\%)$ & $-19.9 \pm 8.2$ & $-15.7 \pm 4.7$ & 0.007 \\
\hline Global RV $\varepsilon_{s y s}(\%)$ & $-16.1 \pm 4.4$ & $-12.9 \pm 2.9$ & 0.000 \\
\hline RV Basal sept TTP (ms) & $339.2 \pm 68.5$ & $359.8 \pm 71.4$ & 0.014 \\
\hline RV Mid sept TTP (ms) & $391.5 \pm 69.3$ & $356.7 \pm 66.1$ & 0.077 \\
\hline RV Apical sept TTP (ms) & $389.2 \pm 97.4$ & $377.3 \pm 71.9$ & 0.869 \\
\hline RV Basal RVFW TTP (ms) & $396 \pm 79.3$ & $366.8 \pm 80.1$ & 0.026 \\
\hline RV Mid RVFW TTP (ms) & $386.7 \pm 72.1$ & $368.8 \pm 80.1$ & 0.003 \\
\hline RV Apical RVFW TTP (ms) & $412.3 \pm 99.9$ & $388.2 \pm 70.6$ & 0.070 \\
\hline Shortest RV TTP (ms) & $326.1 \pm 45.4$ & $314.4 \pm 57.8$ & 0.368 \\
\hline
\end{tabular}




\section{Continued}

\begin{tabular}{cccc}
\hline Longest RV TTP (ms) & $458.1 \pm 78.4$ & $433.1 \pm 61.3$ & 0.070 \\
RV TTP-d (ms) & $132 \pm 76.4$ & $117.2 \pm 70.2$ & 0.145 \\
RV TTP-SD & $46.9 \pm 35.2$ & $40.1 \pm 29.9$ & 0.322 \\
\hline
\end{tabular}

$\mathcal{E}_{\text {sys: }}$ Peak systolic strain; TTP: time to peak systolic strain, TTP-d: electromechanical delay; TTP-SD: standared deviation of mean time to peak.

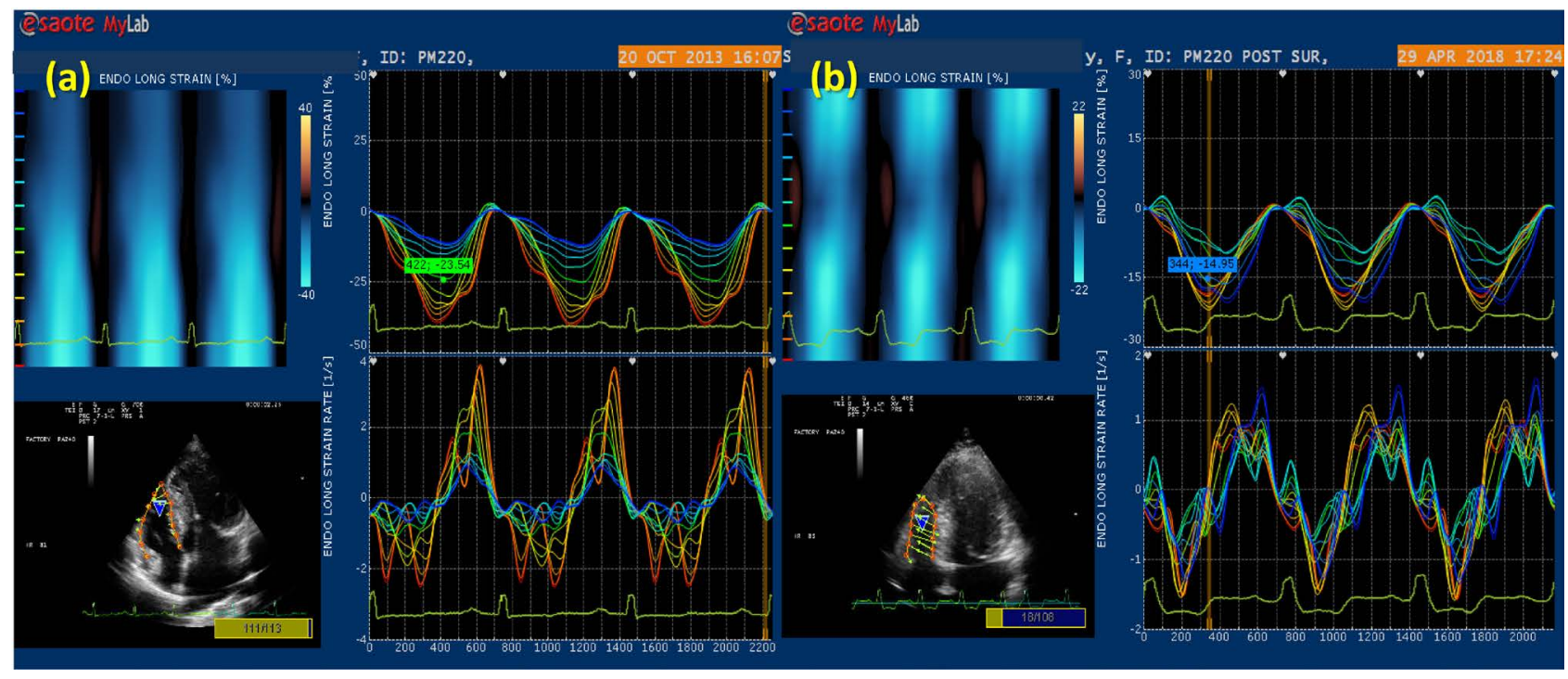

Figure 3. Vector velocity imaging (VVI) of right ventricular (RV) in apical $4 \mathrm{CH}$ view. (a) Before (b) post myectomy, bottom left: RV vector velocity tracking, top left: curved $\mathrm{M}$ mode of septal and RV free wall, top right: RV longitudinal strain curves, bottom right: RV longitudinal strain rate curves. Pre-myectomy GLS was $22.54 \%$ reduced to $14.95 \%$ post myectomy.
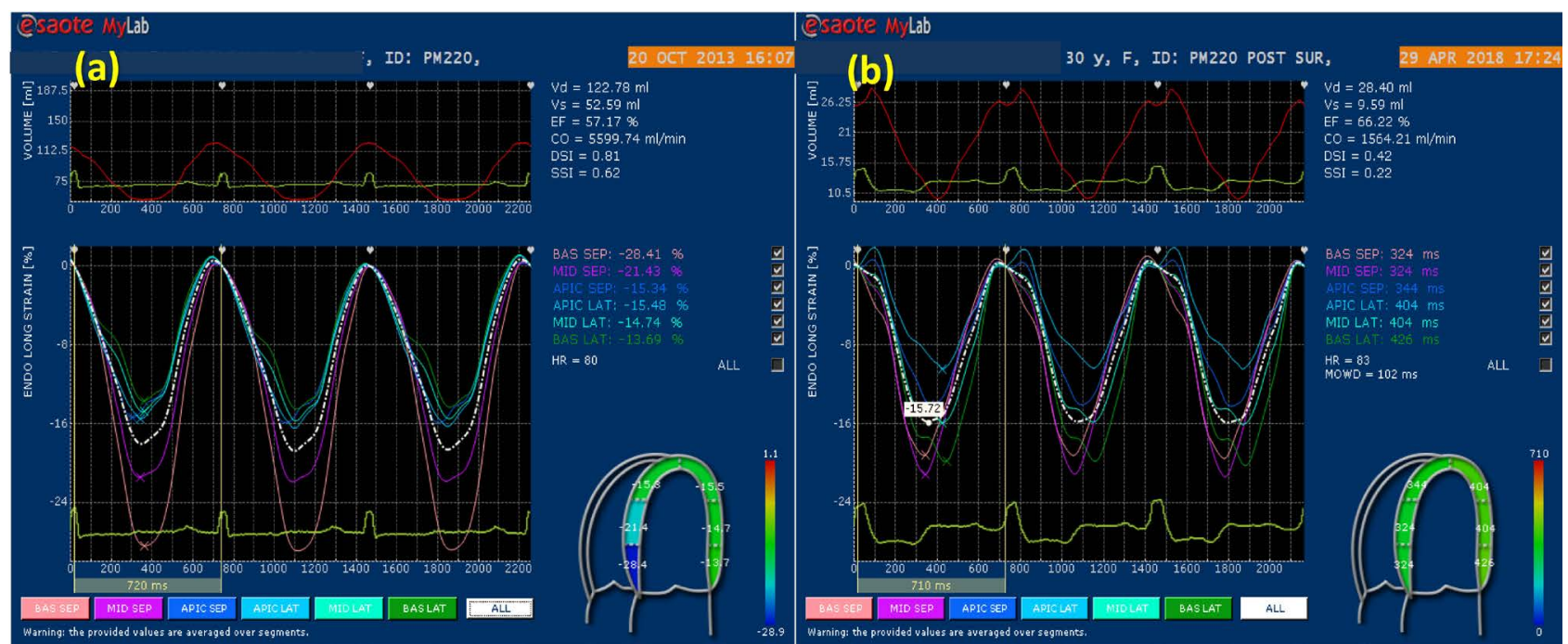

Figure 4. Peak systolic strain curve of septal and right ventricular (RV) free wall segments in hypertrophic cardiomyopathy patient before (a) and after (b) surgical myectomy. Mean value of RV peak systolic strain $\left(\varepsilon_{s y s} \%\right)$ is $-21.24 \%$ before myectomy and reduced to $-15.72 \%$ post myectomy. 

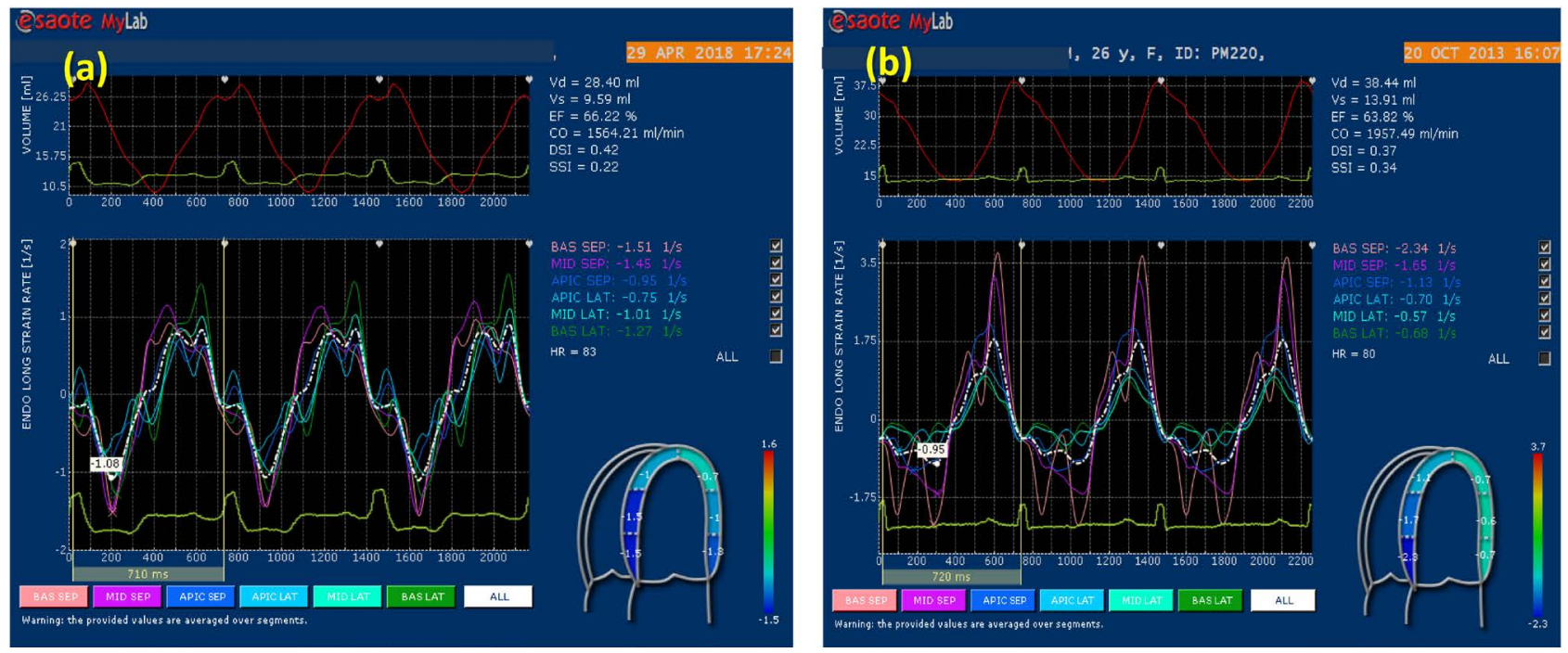

Figure 5. A strain rate curve of septal and right ventricular free wall segments in hypertrophic cardiomyopathy patient before (a) and after (b) surgical myectomy. Mean value of RV peak systolic strain rate $\left(\mathrm{SR}_{\text {sys }}\right)$ is $-1.08 \mathrm{~s}^{-1}$ before myectomy and reduced to $-0.95 \mathrm{~s}^{-1}$ post myectomy.

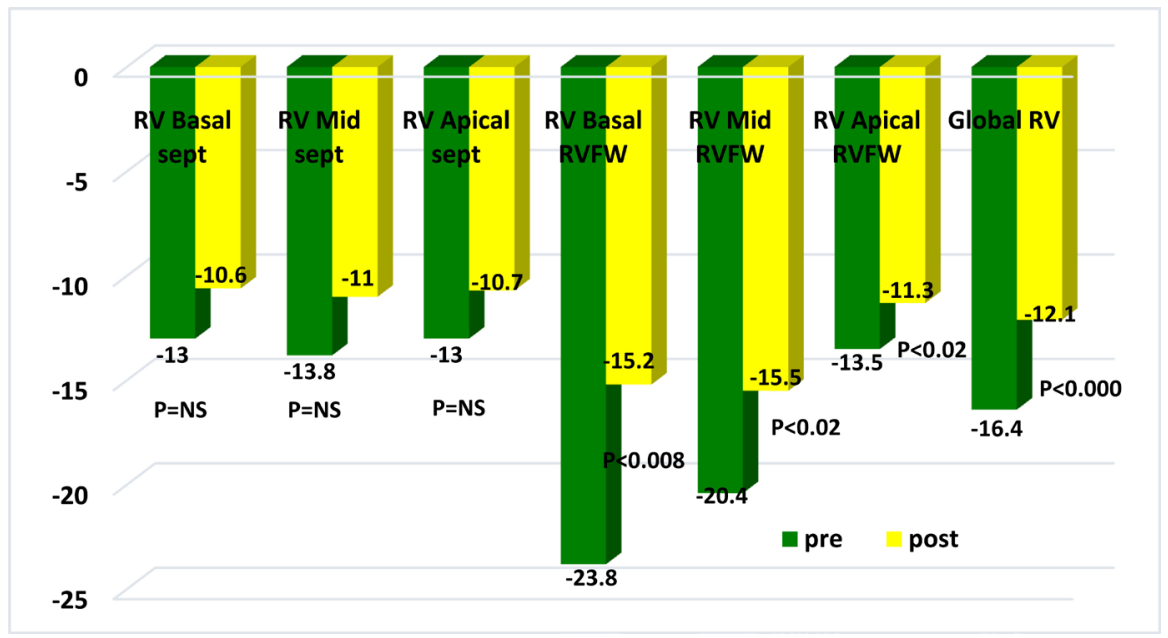

Figure 6. Comparison between RV longitudinal strain $\varepsilon_{s y s}(\%)$ pre- and post-surgical myectomy; RV: right ventricular, $\varepsilon_{\text {sys }}(\%)$ : peak systolic strain, sept: septum, RVFW: right ventricular free wall.

Table 4. Changes in RV strain rate post myectomy.

\begin{tabular}{cccc}
\hline & Pre-myecyomy & Post-myecyomy & P Value \\
\hline $\mathrm{SR}_{\text {sys }}$ Basal sept & $-0.9 \pm 0.5$ & $-0.8 \pm 0.3$ & 0.457 \\
$\mathrm{SR}_{\text {sys }}$ Mid sept & $-0.9 \pm 0.3$ & $-0.7 \pm 0.4$ & 0.141 \\
$\mathrm{SR}_{\text {sys }}$ Apical sept & $-0.8 \pm 0.4$ & $-0.7 \pm 0.4$ & 0.581 \\
$\mathrm{SR}_{\text {sys }}$ Mean sept & $-0.8 \pm 0.4$ & $-0.7 \pm 0.3$ & $\mathbf{0 . 2 7 6}$ \\
$\mathrm{SR}_{\text {sys }}$ Basal RVFW & $-1.6 \pm 1$ & $-1.0 \pm 0.5$ & 0.003 \\
$\mathrm{SR}_{\text {sys }}$ Mid RVFW & $-1.1 \pm 0.6$ & $-0.9 \pm 0.5$ & 0.066 \\
\hline
\end{tabular}




\section{Continued}

\begin{tabular}{|c|c|c|c|}
\hline $\mathrm{SR}_{\text {sys }}$ Apical RVFW & $-0.8 \pm 0.4$ & $-0.8 \pm 0.4$ & 0.944 \\
\hline $\mathrm{SR}_{\text {sys }}$ Mean RVFW & $-1.2 \pm 0.7$ & $-0.9 \pm 0.4$ & 0.028 \\
\hline $\mathrm{SR}_{\text {sys }}$ Global & $-1.0 \pm 0.4$ & $-0.8 \pm 0.3$ & 0.036 \\
\hline $\mathrm{SR}_{\mathrm{e}}$ Basal sept & $0.9 \pm 0.4$ & $0.5 \pm 0.3$ & 0.002 \\
\hline $\mathrm{SR}_{\mathrm{e}}$ Mid sept & $0.8 \pm 0.3$ & $0.5 \pm 0.3$ & 0.006 \\
\hline $\mathrm{SR}_{\mathrm{e}}$ Apical sept & $0.8 \pm 0.4$ & $0.5 \pm 0.4$ & 0.009 \\
\hline $\mathrm{SR}_{\mathrm{e}}$ Mean sept & $0.8 \pm 0.3$ & $0.5 \pm 0.3$ & 0.002 \\
\hline $\mathrm{SR}_{\mathrm{e}}$ Basal RVFW & $1.5 \pm 1.2$ & $0.8 \pm 0.7$ & 0.026 \\
\hline $\mathrm{SR}_{\mathrm{e}} \mathrm{Mid} \mathrm{RVFW}$ & $1.1 \pm 0.9$ & $0.7 \pm 0.5$ & 0.134 \\
\hline $\mathrm{SR}_{\mathrm{e}}$ Apical RVFW & $0.7 \pm 0.4$ & $0.7 \pm 0.4$ & 0.573 \\
\hline $\mathrm{SR}_{\mathrm{e}}$ Mean RVFW & $1.0 \pm 0.8$ & $0.8 \pm 0.3$ & 0.144 \\
\hline $\mathrm{SR}_{\mathrm{e}}$ Global RV & $0.9 \pm 0.4$ & $0.7 \pm 0.3$ & 0.019 \\
\hline $\mathrm{SR}_{\mathrm{a}}$ Basal sept & $0.8 \pm 0.4$ & $0.5 \pm 0.3$ & 0.004 \\
\hline $\mathrm{SR}_{\mathrm{a}}$ Mid sept & $0.7 \pm 0.3$ & $0.5 \pm 0.3$ & 0.007 \\
\hline $\mathrm{SR}_{\mathrm{a}}$ Apical sept & $0.7 \pm 0.3$ & $0.5 \pm 0.4$ & 0.036 \\
\hline $\mathrm{SR}_{\mathrm{a}}$ Mean sept & $0.7 \pm 0.3$ & $0.5 \pm 0.3$ & 0.007 \\
\hline $\mathrm{SR}_{\mathrm{a}}$ Basal RVFW & $1.2 \pm 0.8$ & $0.9 \pm 0.8$ & 0.032 \\
\hline $\mathrm{SR}_{\mathrm{a}}$ Mid RVFW & $1.0 \pm 0.5$ & $0.7 \pm 0.4$ & 0.083 \\
\hline $\mathrm{SR}_{\mathrm{a}}$ Apical RVFW & $0.6 \pm 0.3$ & $0.5 \pm 0.3$ & 0.480 \\
\hline $\mathrm{SR}_{\mathrm{a}}$ Mean RVFW & $1.1 \pm 0.6$ & $0.9 \pm 0.5$ & 0.154 \\
\hline $\mathrm{SR}_{\mathrm{a}}$ Global RV & $0.9 \pm 0.6$ & $0.6 \pm 0.3$ & 0.034 \\
\hline
\end{tabular}

Comparison between Right ventricular strain rate parameters pre- and post-myectomy; RV: right ventricle, sept.: septum, RVFW: right ventricular free wall, SRsys: peak systolic strain rate, SRe: early diastolic strain rate, SRa: atrial diastolic strain rate.

there was significant reduction in segmental and global $\mathrm{RV} \mathrm{SR}_{\mathrm{sys}}, \mathrm{SR}_{\mathrm{e}}$, and $\mathrm{SR}_{\mathrm{a}}$ $(-13.4 \pm 4.6$ vs $-19.8 \pm 9.4 ; \mathrm{p} 0.002)$, and $(-12.1 \pm 2.8$ vs $-16.4 \pm 4.7 ; \mathrm{p}: 0.0001)$ respectively.

\subsection{Strain}

LV septal longitudinal strain was significantly attenuated post surgical myectomy $(\mathrm{P}<0.001)$ as well as anterior and inferior LV walls strain $\left(\varepsilon_{s y s} \%\right)$ showed significant reduction $(\mathrm{P}<0.05,<0.02$ respectively. Post-myectomy reduction in LV global longitudinal strain was strongly evident compared with corresponding values before surgery $(\mathrm{P}<0.001)$.

Comparison between LV longitudinal strain $\varepsilon_{s y s} \%$ pre and post-surgical myectomy in the study population are summarized in Table 5 and Figure 7. 
Table 5. Changes in segmental and global left ventricular peak systolic strain post-myectomy.

\begin{tabular}{|c|c|c|c|}
\hline & Pre-myecyomy & Post-myecyomy & $P$ value \\
\hline LV Basal sept $\varepsilon_{s y s}(\%)$ & $-9.2 \pm 3.6$ & $-6.3 \pm 2.2$ & 0.000 \\
\hline LV Mid sept $\varepsilon_{s y s}(\%)$ & $-11.3 \pm 4.0$ & $-7.2 \pm 2.4$ & 0.000 \\
\hline LV Apical sept $\varepsilon_{s y s}(\%)$ & $-13.2 \pm 5.6$ & $-8.4 \pm 3.7$ & 0.000 \\
\hline Mean sept $\varepsilon_{s y s}(\%)$ & $-11.2 \pm 3.2$ & $-7.3 \pm 2.0$ & 0.000 \\
\hline LV Basal lateral $\varepsilon_{s y s}(\%)$ & $-15.4 \pm 5.5$ & $-15.9 \pm 6.4$ & 0.734 \\
\hline LV Mid lateral $\varepsilon_{s y s}(\%)$ & $-12.8 \pm 4.5$ & $-12.4 \pm 4.9$ & 0.659 \\
\hline LV Apical lateral $\varepsilon_{s y s}(\%)$ & $-10.5 \pm 4.7$ & $-9.1 \pm 3.9$ & 0.134 \\
\hline Mean lateral $\varepsilon_{s y s}(\%)$ & $-12.9 \pm 3.4$ & $-12.5 \pm 4.3$ & 0.607 \\
\hline LV Basal Anterior $\varepsilon_{s y s}(\%)$ & $-13.3 \pm 8.3$ & $-9.0 \pm 5.1$ & 0.007 \\
\hline LV Mid Anterior $\varepsilon_{s y s}(\%)$ & $-11.3 \pm 7.2$ & $-9.2 \pm 3.7$ & 0.118 \\
\hline LV Apical Anterior $\varepsilon_{s y s}(\%)$ & $-9.4 \pm 5.9$ & $-8.8 \pm 3.3$ & 0.664 \\
\hline Mean Anterior $\mathcal{E}_{s y s}(\%)$ & $-11.3 \pm 6.3$ & $-9.0 \pm 3.5$ & 0.052 \\
\hline LV Basal Inferior $\varepsilon_{s y s}(\%)$ & $-11.8 \pm 4.4$ & $-8.9 \pm 2.9$ & 0.005 \\
\hline LV Mid Inferior $\mathcal{E}_{s y s}(\%)$ & $-9.5 \pm 5.6$ & $-8.1 \pm 2.2$ & 0.240 \\
\hline LV Apical Inferior $\varepsilon_{s y s}(\%)$ & $-11.2 \pm 4.8$ & $-9.1 \pm 3.7$ & 0.028 \\
\hline Mean Inferior $\varepsilon_{s y s}(\%)$ & $-10.9 \pm 3.2$ & $-8.7 \pm 2.3$ & 0.002 \\
\hline Global LV $\varepsilon_{s y s}(\%)$ & $-11.6 \pm 2.8$ & $-9.4 \pm 2.2$ & 0.000 \\
\hline
\end{tabular}

LV: left ventricular, $\varepsilon_{s y s}(\%)$ : peak longitudinal strain, sept: septum.

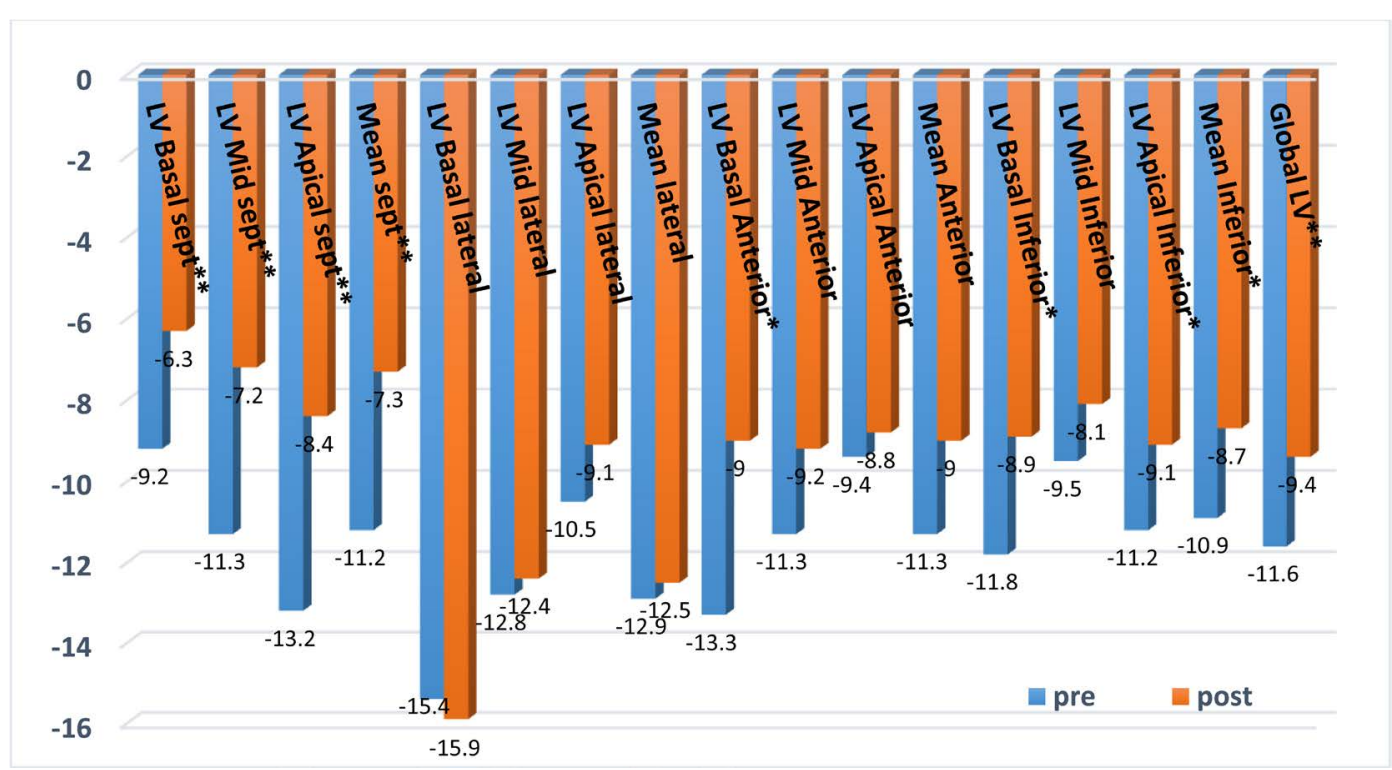

LV: left ventricular, $\varepsilon_{s y s}(\%):$ longitudinal strain percent, sept: septum ${ }^{\star}: \mathrm{p}<0.05,{ }^{*}: \mathrm{p}<0.0001$

Figure 7. Comparison between LV longitudinal strain $\varepsilon_{s y s}(\%)$ pre- and post-surgical myectomy. 


\subsection{Univariate Analysis of RV Strain Indexes (Table 6, Figure 8)}

In HCM, The magnitude of reduction in cardiac mechanics was tested against the pre and post deformation parameters and cardiac phenotype as shown in Table 6. $\Delta \mathrm{RV}$ septum $\varepsilon_{s y s}(\%)$ was correlated directly to pre-LVOT gradient $(\mathrm{P}<$ $0.03)$ and post-RVTTP-SD $(\mathrm{P}<0.04)$. $\Delta$ Global RV $\varepsilon_{s y s}(\%)$ was directly correlated to LV MWT $(\mathrm{P}<0.01)$ and negatively correlated to LV EF \% $(\mathrm{P}<0.03)$. $\triangle \mathrm{RV}$ TTP SD was directly correlated to pre-RV TTP SD $(\mathrm{P}<0.001)$ and negatively correlated to LV EF \% $(\mathrm{P}<0.03)$ and $\mathrm{LA}$ volume $(\mathrm{P}<0.003) . \Delta \mathrm{RV}$ septum $\mathrm{SR}_{\text {sys }}$ was directly correlated to RV TTP post myectomy $(\mathrm{P}<0.03)$ and negatively correlated to LVOT gradient $(\mathrm{P}<0.028)$ while $\Delta$ Global $\mathrm{RV} \mathrm{SR}$ sys was directly

Table 6. Univariate analysis of ECHO parameters of RV mechanics post-myectomy.

\begin{tabular}{|c|c|c|c|c|c|c|c|c|c|c|c|c|c|c|c|c|}
\hline & & $\begin{array}{c}\text { Global LV } \\
\varepsilon_{s y s}(\%) \\
\text { (Pre } \\
\text { myectomy) }\end{array}$ & $\begin{array}{c}\text { Global LV } \\
\varepsilon_{s y s}(\%) \\
\text { (Post } \\
\text { myectomy) }\end{array}$ & $\begin{array}{c}\text { RV } \\
\text { TTP-SD } \\
\text { (Pre } \\
\text { myectomy) }\end{array}$ & $\begin{array}{c}\text { RV } \\
\text { TTP-SD } \\
\text { (Post } \\
\text { myectomy) }\end{array}$ & $\begin{array}{l}\text { RV TTP-d } \\
\quad \text { (Pre } \\
\text { myectomy) }\end{array}$ & $\begin{array}{l}\text { RV TTP-d } \\
\quad \text { (Post } \\
\text { myectomy) }\end{array}$ & $\begin{array}{l}\text { Age } \\
\text { (Ys) }\end{array}$ & $\begin{array}{l}\text { Grade } \\
\text { of MR }\end{array}$ & $\begin{array}{c}\text { LA } \\
\text { volume }\end{array}$ & MWT & $\begin{array}{c}\text { LVOT } \\
\text { PG }\end{array}$ & PAP & EDD & FS $\%$ & $\mathrm{EF} \%$ \\
\hline \multirow{2}{*}{$\begin{array}{c}\Delta \mathrm{RV} \\
\text { septum } \mathcal{E}_{s y s} \\
(\%)\end{array}$} & $\mathrm{R}$ & 0.06 & 0.06 & -0.18 & -0.4 & 0.07 & 0.28 & -0.05 & -0.34 & -0.24 & -0.13 & -0.43 & -0.26 & 0.28 & -0.03 & 0.02 \\
\hline & $\mathrm{P}$ & 0.764 & 0.784 & 0.38 & 0.048 & 0.75 & 0.172 & 0.795 & 0.096 & 0.255 & 0.541 & 0.03 & 0.213 & 0.177 & 0.876 & 0.93 \\
\hline \multirow{2}{*}{$\begin{array}{c}\Delta \text { Global } \\
\operatorname{RV} \varepsilon_{s y s}(\%)\end{array}$} & $\mathrm{R}$ & 0.26 & 0.13 & -0.08 & -0.02 & 0.02 & 0.29 & 0.27 & -0.01 & 0.15 & 0.46 & -0.15 & -0.25 & -0.03 & -0.08 & -0.43 \\
\hline & $\mathrm{P}$ & 0.204 & 0.521 & 0.699 & 0.933 & 0.936 & 0.158 & 0.186 & 0.957 & 0.473 & 0.019 & 0.481 & 0.225 & 0.87 & 0.692 & 0.034 \\
\hline \multirow{2}{*}{$\begin{array}{c}\Delta \mathrm{RV} \text { TTP } \\
\mathrm{SD}\end{array}$} & $\mathrm{R}$ & -0.26 & -0.03 & -0.62 & 0.48 & -0.34 & -0.29 & -0.03 & 0.15 & -0.586 & -0.1 & -0.01 & 0.06 & 0.11 & 0.07 & -0.44 \\
\hline & $\mathrm{P}$ & 0.203 & 0.872 & 0.001 & 0.014 & 0.094 & 0.158 & 0.904 & 0.467 & 0.003 & 0.628 & 0.963 & 0.76 & 0.601 & 0.73 & 0.028 \\
\hline \multirow{2}{*}{$\begin{array}{c}\Delta \mathrm{RV} \text { TTP } \\
\text { delay }\end{array}$} & $\mathrm{R}$ & -0.26 & 0.02 & -0.63 & 0.44 & -0.26 & -0.29 & -0.06 & 0.12 & -0.611 & -0.15 & -0.04 & 0.09 & 0.15 & -0.01 & -0.32 \\
\hline & $\mathrm{P}$ & 0.209 & 0.917 & 0.001 & 0.029 & 0.217 & 0.153 & 0.766 & 0.577 & 0.001 & 0.469 & 0.846 & 0.66 & 0.469 & 0.964 & 0.118 \\
\hline $\begin{array}{c}\Delta \mathrm{RV} \\
\text { septum }\end{array}$ & $\mathrm{R}$ & -0.03 & 0.09 & -0.11 & -0.38 & 0.22 & 0.44 & -0.04 & -0.05 & 0.01 & -0.1 & -0.44 & -0.11 & 0.01 & -0.16 & 0.06 \\
\hline $\mathrm{SR}_{\text {sys }}$ & $P$ & 0.903 & 0.669 & 0.595 & 0.059 & 0.294 & 0.03 & 0.866 & 0.801 & 0.978 & 0.628 & 0.028 & 0.594 & 0.949 & 0.454 & 0.788 \\
\hline \multirow{2}{*}{$\begin{array}{l}\Delta \text { Global } \\
\mathrm{RV} \mathrm{SR}_{\mathrm{sys}}\end{array}$} & $\mathrm{R}$ & -0.16 & -0.18 & 0.11 & 0.17 & -0.11 & 0.04 & -0.46 & 0.04 & -0.09 & 0.08 & -0.11 & -0.26 & -0.25 & -0.09 & -0.38 \\
\hline & $\mathrm{P}$ & 0.458 & 0.377 & 0.613 & 0.417 & 0.596 & 0.831 & 0.02 & 0.861 & 0.673 & 0.709 & 0.612 & 0.213 & 0.223 & 0.669 & 0.061 \\
\hline \multirow{2}{*}{$\begin{array}{c}\Delta \text { septum } \\
\mathrm{SR}_{\mathrm{e}}\end{array}$} & $\mathrm{R}$ & 0.13 & 0.12 & -0.21 & 0.02 & 0.02 & -0.23 & 0.08 & -0.26 & -0.02 & 0.07 & 0.1 & -0.09 & 0.41 & -0.12 & 0.06 \\
\hline & $\mathrm{P}$ & 0.549 & 0.58 & 0.32 & 0.91 & 0.937 & 0.259 & 0.709 & 0.207 & 0.912 & 0.728 & 0.641 & 0.67 & 0.039 & 0.561 & 0.775 \\
\hline $\begin{array}{l}\Delta \text { Global } \\
\text { RV SR }_{\mathrm{e}}\end{array}$ & $\mathrm{P}$ & 0.536 & 0.709 & 0.912 & 0.638 & 0.394 & 0.734 & 0.186 & 0.342 & 0.616 & 0.994 & 0.683 & 0.973 & 0.024 & 0.893 & 0.207 \\
\hline \multirow{2}{*}{$\begin{array}{c}\Delta \text { septum } \\
\mathrm{SR}_{\mathrm{a}}\end{array}$} & $\mathrm{R}$ & -0.14 & 0.07 & 0.04 & 0.12 & 0.3 & 0.36 & -0.05 & 0.16 & 0.25 & 0.07 & 0.62 & 0.61 & -0.04 & 0.21 & 0.27 \\
\hline & $\mathrm{P}$ & 0.516 & 0.723 & 0.847 & 0.553 & 0.146 & 0.074 & 0.815 & 0.454 & 0.235 & 0.728 & 0.001 & 0.001 & 0.851 & 0.317 & 0.194 \\
\hline \multirow{2}{*}{$\begin{array}{l}\Delta \text { Global } \\
\mathrm{RV} \mathrm{SR}_{\mathrm{a}}\end{array}$} & $\mathrm{R}$ & -0.13 & 0.01 & 0.12 & 0.28 & 0.34 & 0.32 & -0.02 & 0.13 & 0.14 & 0.1 & 0.58 & 0.57 & -0.06 & 0.29 & 0.39 \\
\hline & $\mathrm{P}$ & 0.524 & 0.944 & 0.579 & 0.172 & 0.095 & 0.123 & 0.917 & 0.55 & 0.494 & 0.631 & 0.002 & 0.003 & 0.787 & 0.162 & 0.056 \\
\hline
\end{tabular}

$\varepsilon_{\text {sys }}$ Peak systolic strain; TTP: time to peak systolic strain, TTP-d: electromechanical delay; TTP-SD: standared deviation of mean time to peak. RVFW: right ventricular free wall, SRsys: peak systolic strain rate, SRe: early diastolic strain rate, SRa: atrial diastolic strain rate. 


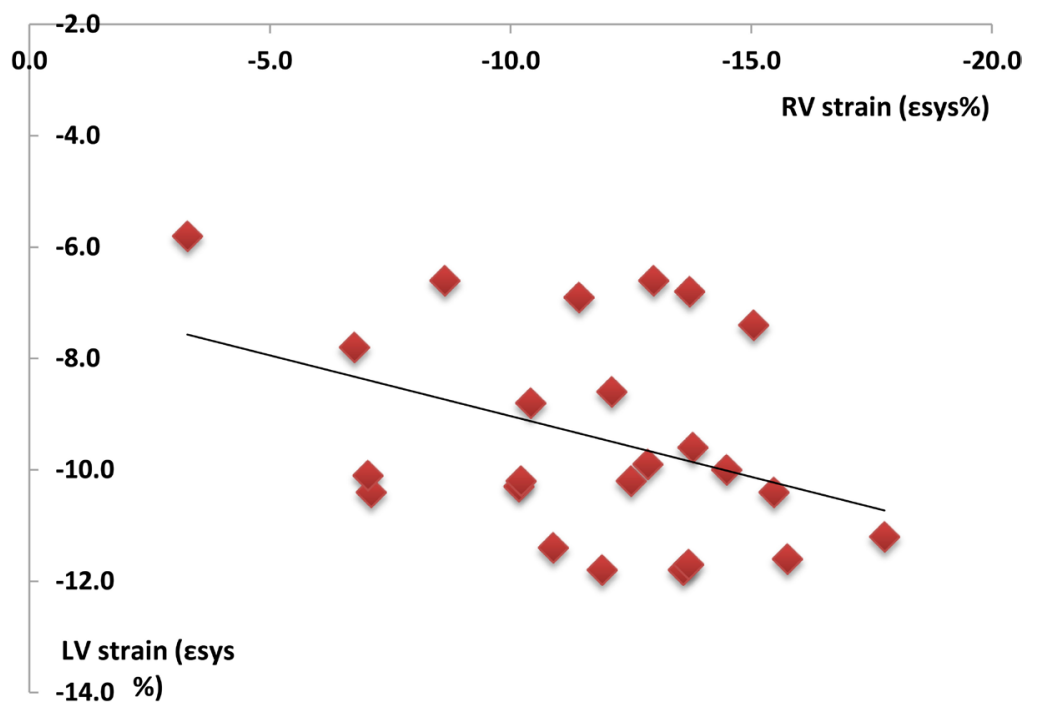

Figure 8. Relationship of RV strain to LV strain post myectomy.

correlated to age $(\mathrm{P}<0.02)$. Regarding diastolic parameters, $\Delta$ septum $\mathrm{SR}_{e}$ and $\Delta$ global $\mathrm{SR}_{\mathrm{e}}$ were inversely correlated with end diastolic diameter $(\mathrm{P}<0.04$ and $<0.03)$ respectively. Meanwhile, reduction in atrial contractile function $(\Delta$ septum $\mathrm{SR}_{\mathrm{a}}$ and $\Delta$ global $\left.\mathrm{SR}_{\mathrm{a}}\right)$ was directly correlated to LVOT gradient $(\mathrm{P}<0.001$, $<0.002)$ and PAP $(\mathrm{P}<0.001,<0.003)$ respectively.

\subsection{Relationship of RV Deformation to LV Strain}

There was significant reduction in longitudinal strain at the myectomy segments, averaged IVS and global LV longitudinal strain, which remained lower than normal, Pearson correlation coefficient of global RV $\left(\varepsilon_{s y s} \%\right)$ showed modest correlation to LV $\varepsilon_{\text {sys }} \%(\mathrm{r}=0.39, \mathrm{P}<0.047)$ postmyectomy. However, no significant correlation was detected between other RV deformation parameters and $\mathrm{LV} \boldsymbol{\varepsilon}_{\text {sys }} \%, \mathrm{RV} \mathrm{SR}$ sys $(\mathrm{r}=0.31 . \mathrm{P}<0.07)$, RV SR $\mathrm{e}(\mathrm{r}=0.098, \mathrm{P}=0.84)$ or $\mathrm{RV} \mathrm{SR}_{\mathrm{a}}(\mathrm{r}=0.198, \mathrm{P}=0.63)$ post myectomy.

\section{Discussion}

In the current study both regional and global RV function, as measured by $2 \mathrm{D}$ strain imaging, evidently decline post-surgical myectomy. Despite the improvement of NYHA class, severity of mitral regurge, reduction of E/E' and LV mass, LV deformation showed marked deterioration. $\mathrm{RV}$ deformational decline is predominantly associated with impaired LV longitudinal strain and correlated to the severity of LV hypertrophy. Impaired RV diastolic function is correlated to severity of LVOT obstruction and PAP, while RV intraventricular dyssynchrony is mainly related to LA volume and LV EF \%.

\subsection{RV Strain Using Speckle Tracking as a Measure of Cardiac Function}

GLS assessed using speckle-tracking echocardiography (STE) is an emerging 
technique for detecting and quantifying subtle disturbances in cardiac mechanics. GLS reflects the longitudinal contraction of the myocardium and its accuracy has been validated against tagged magnetic resonance imaging (MRI) [13]. This method is operator independent, more reproducible than conventional parameters, easily measured and integrated to standard echocardiogram method [14]. In the general population and patients with heart failure, GLS was shown to be a superior predictor of cardiac events and all-cause mortality compared to EF [13] [15]. More recently, GLS was found to be a robust prognostic marker following cardiac surgery [16] [17] [18]. GLS predicted postoperative mortality, myocardial dysfunction, and the need for prolonged inotropic support in cardiac surgical patients, though GLS was measured preoperatively by TTE in many recent research studies [19] [20].

In the present study, we characterized the change in RV function following myectomy using established deformational RV 2D strain techniques.

\subsection{RV Function Following Cardiac Surgery}

While RV function is an important determinant of cardiac surgical outcomes, there are few known predictors of postoperative dysfunction. Studies demonstrate that RV fractional area change (FAC) $<35 \%$ is associated with the greatest risk of postoperative mortality [21] [22] and that among patients with preserved RV FAC, those with abnormal longitudinal strain are at higher risk for post-operative mortality [23]. Despite our finding that all patients have symptomatic improvement, RV longitudinal indices including strain and strain rate showed significant deterioration post myectomy and after few months post-operative that ensure patient recovery, which might predict persistent dysfunction.

Using the feature tracking VVI, RV global longitudinal strain not only measures contraction but is also able to reflect interstitial myocardial changes such as fibrosis, which are one of main histopathological changes following cardiac surgery [24] [25].

\subsection{Mechanisms of Post Myectomy RV Dysfunction}

In the present study $2 \mathrm{D}$ strain imaging was used to characterize the changes in RV mechanics post myectomy. Both segmental and global RV mechanics showed marked decline especially with proved RV dysfunction before myectomy. A variety of hypotheses have been proposed to explain the reduction of RV function even following uncomplicated cardiac surgery. Such theories include pericardial disruption, injury to the right atrium from venous cannulation, ischemic injury to the RV due to incomplete myocardial protection while on cardiopulmonary bypass, and formation of adhesions between the right ventricle and surrounding mediastinal tissues [26] [27]. These factors are superimposed on already impaired RV dysfunction due to hypertrophic myopathic process.

The reduced RV contractile function in our study was associated with the 
reduction in LV function. Generally, LV function may be impaired by cardioplegia-induced myocardial arrest and ischemia-reperfusion injury, especially in those patients with hypertrophic cardiomyopathy. Alternatively, LV function may improve on long term follow up when afterload is reduced following myectomy and resolution of LVOTO. The right ventricle (RV), however, experiences cardioplegic arrest without benefiting from improved loading conditions [28].

Unlike patients with aortic valve replacement, where LV function improved after replacement of a stenotic aortic valve as demonstrated by improved longitudinal strain; LV function post myectomy is deteriorated. In the current study, $80 \%$ developed complete LBBB and 4\% developed RBBB after surgery which might be the real underlying cause of LV mechanical dysfunction and electromechanical dyssynchrony detected in HCM patients post myectomy [28] [29].

Furthermore, in HCM patients another explanation can be added which is replacement fibrosis of LV myocardial segments following surgical excision of septal segments which is completely different from valve replacement without interference in myocardial architechture or myocardial vasculature.

To our knowledge, this is the most inclusive study of changes in RV systolic function and diastolic function postoperatively with correlation of changes in $2 \mathrm{D}$ strain imaging with regional and global LV strain. We demonstrate that postoperative RV systolic function significantly declines across all parameters few months following surgery; in particular, GLS which significantly predict persistant subnormal RV function that predicts poor outcome. Meanwhile, our measurements were taken longtime after surgery, which suggests that changes in RV indices were due to both functional and geometric changes.

Unlike Doppler interrogation, strain measured by 2D is angle independent. Therefore, it may be more accurate and easily applied than tissue Doppler interrogation (TDI). However, 2D STE is dependent on image quality and has lower temporal resolution than TDI. While 2D STE is used to demonstrate subclinical LV dysfunction independent of changes in ejection fraction, it is not yet routinely used to characterize RV dysfunction [30] [31]. Our study demonstrates that it is feasible to use strain as a comparable index to predict RV function in the postoperative period [32] [33].

$\mathrm{RV}$ strain may be an important index to detect RV dysfunction and provide postoperative risk stratification that might have important clinical implications. Nevertheless, it remains unclear precisely when and why these indices of RV systolic function are reduced.

\subsection{Study Limitations}

There were several limitations to this study. First, the RV is difficult to image in its entirety in one view and is difficult to model. A 3D RV function analysis, such as $3 \mathrm{D}$ speckle tracking, might have been superior to $2 \mathrm{D}$ only. Second Subtle changes in other areas of the RV myocardium might have been detected. Third, 
only longitudinal strain was analyzed, whereas analysis of radial or circumferential strain might have provided some additional clues into whether contraction patterns change in response to myectomy or surgery itself. Additionally, patients did not undergo long-term follow-up with a transthoracic echocardiogram to assess whether any aspect of recovery was apparent at a later date or whether any changes in contraction patterns evolved in the face of a persistent depression of LV longitudinal strain.

\section{Conclusion}

Surgical myectomy deteriorates RV function, as measured by two-dimensional myocardial strain imaging. The magnitude of RV function decline is proportionate to LV dyfunction and severity of cardiac phenotype. Worsening of RV strain, however, and its clinical implications in HCM population require further exploration. The feasibility, high reproducibility, and validated reference values of deformation imaging may support more routine post operative use of this modality in the TTE examination of HCM patients.

\section{Conflicts of Interest}

The authors declare no conflicts of interest regarding the publication of this paper.

\section{References}

[1] Maron, B.J. (1997) Hypertrophic Cardiomyopathy. The Lancet, 350, 127-133. https://doi.org/10.1016/S0140-6736(97)01282-8

[2] Maron, M.S., Hauser, H.H., Dubrow, E., Horst, T.A., Kissinger, K.V., et al. (2007) Right Ventricular Involvement in Hypertrophic Cardiomyopathy. The American Journal of Cardiology, 100, 1293-1298. https://doi.org/10.1016/j.amjcard.2007.05.061

[3] Solomon, Z., Breton, C., Rowin, E.J., Maron, B.J., Maron, M.S., Chen, F.Y. and Rastegar, H. (2018) Surgical Approaches to Hypertrophic Obstructive Cardiomyopathy. Seminars in Thoracic and Cardiovascular Surgery, 30, 125-128. https://doi.org/10.1053/j.semtcvs.2018.02.034

[4] Ommen, S.R., Maron, B.J., Olivotto, I., Maron, M.S., Cecchi, F., Betocchi, S., et al. (2005) Long-Term Effects of Surgical Septal Myectomy on Survival in Patients with Obstructive Hypertrophic Cardiomyopathy. Journal of the American College of Cardiology, 46, 470-476. https://doi.org/10.1016/j.jacc.2005.02.090

[5] Ogiwara, F., Koyama, J., Ikeda, S., Kinoshita, O. and Falk, R.H. (2005) Comparison of the Strain Doppler Echocardiographic Features of Familial Amyloid Polyneuropathy (FAP) and Light-Chain Amyloidosis. The American Journal of Cardiology, 95, 538-540. https://doi.org/10.1016/j.amjcard.2004.10.029

[6] Mor-Avi, V., Lang, R.M., Badano, L.P., Belohlavek, M., Cardim, N.M., Derumeaux, G., et al. (2011) Current and Evolving Echocardiographic Techniques for the Quantitative Evaluation of Cardiac Mechanics: ASE/EAEconsensus Statement on Methodology and Indications Endorsed by the Japanese Society of Echocardiography. Journal of the American Society of Echocardiography, 24, 277-313. https://doi.org/10.1016/j.echo.2011.01.015 
[7] D’Andrea, A., Caso, P., Bossone, E., Scarafile, R., Riegler, L., Di Salvo, G., et al (2010) Right Ventricular Myocardial Involvement in Either Physiological or Pathological Left Ventricular Hypertrophy: An Ultrasound Speckle-Tracking Two-Dimensional Strain Analysis. European Journal of Echocardiography, 11, 492-500. https://doi.org/10.1093/ejechocard/jeq007

[8] Zemanek, D., Tomasov, P., Prichystalova, P., Linhartova, K. and Veselka, J. (2010) Evaluation of the Right Ventricular Function in Hypertrophic Obstructive Cardiomyopathy: A Strain and Tissue Doppler Study. Physiological Research, 59, 697-702.

[9] Badran, H.M., Faheem, N., Soliman, M., Hamdy, M. and Yacoub, M. (2019) Comparison of Vector Velocity Imaging and Three-Dimensional Speckle Tracking Echocardiography for Assessment of Left Ventricular Longitudinal Strain in Hypertrophic Cardiomyopathy. Global Cardiology Science and Practice, 2019, 6. https://doi.org/10.21542/gcsp.2019.6

[10] Bussadori, C., Moreo, A., Di Donato, M., De Chiara, B., Negura, D., Dall'Aglio, E., Lobiati, E., Chessa, M., Arcidiacono, C., Dua, J.S., Mauri, F. and Carminati, M. (2009) A New 2D-Based Method for Myocardial Velocity Strain and Strain Rate Quantification in a Normaladult and Paediatric Population: Assessment of Reference Values. Cardiovascular Ultrasound, 13, 7-8. https://doi.org/10.1186/1476-7120-7-8

[11] Williams, L.K., Urbano-Moral, J.A., Rowin, E.J., Jamorski, M., Bruchal-Garbicz, B., Carasso, S., Pandian, N.G., Maron, M.S. and Rakowski, H. (2013) Velocity Vector Imaging in the Measurement of Left Ventricular Myocardial Mechanics on Cardiac Magnetic Resonance Imaging: Correlations with Echocardiographically Derived Strain Values. Journal of the American Society of Echocardiography, 26, 1153-1162. https://doi.org/10.1016/j.echo.2013.06.008

[12] Al Kindi, H.N. and Yacoub, M.H. (2018) Surgical Myectomy: Rationale and Personalized Technique. Global Cardiology Science and Practice, 2018, 35.

https://doi.org/10.21542/gcsp.2018.35

[13] Amundsen, B.H., Helle-Valle, T., Edvardsen, T., Torp, H., Crosby, J., Lyseggen, E., et al. (2006) Noninvasive Myocardial Strain Measurement by Speckle Tracking Echocardiography: Validation against Sonomicrometry and Tagged Magnetic Resonance Imaging. Journal of the American College of Cardiology, 47, 789-793. https://doi.org/10.1016/j.jacc.2005.10.040

[14] Belghitia, H., Brette, S., Lafitte, S., Reant, P., Picard, F., Serri, K., et al. (2008) Automated Function Imaging: A New Operator-Independent Strain Method for Assessing Left Ventricular Function. Archives of Cardiovascular Diseases, 101, 163-169. https://doi.org/10.1016/S1875-2136(08)71798-4

[15] Cho, G.Y., Marwick, T.H., Kim, H.S., Kim, M.K., Hong, K.S. and Oh, D.J. (2009) Global 2-Dimensional Strain as a New Prognosticator in Patients with Heart Failure. Journal of the American College of Cardiology, 54, 618-624. https://doi.org/10.1016/j.jacc.2009.04.061

[16] Stanton, T., Leano, R. and Marwick, T.H. (2009) Prediction of All-Cause Mortality from Global Longitudinal Speckle Strain: Comparison with Ejection Fraction and Wall Motion Scoring. Circulation: Cardiovascular Imaging, 2, 356-364. https://doi.org/10.1161/CIRCIMAGING.109.862334

[17] Ternacle, J., Berry, M., Alonso, E., Kloeckner, M., Couetil, J.P., Rande, J.L., Gueret, P., Monin, J.L. and Lim, P. (2013) Incremental Value of Global Longitudinal Strain for Predicting Early Outcome after Cardiac Surgery. European Heart JournalCardiovascular Imaging, 14, 77-84. https://doi.org/10.1093/ehjci/jes156

[18] Saito, M., Okayama, H., Yoshii, T., Higashi, H., Morioka, H., Hiasa, G., et al. (2012) 
Clinical Significance of Global Two-Dimensional Strain as a Surrogate Parameter of Myocardial Fibrosis and Cardiac Events in Patients with Hypertrophic Cardiomyopathy. European Heart Journal-Cardiovascular Imaging, 13, 617-623.

https://doi.org/10.1093/ejechocard/jer318

[19] Bartko, P.E., Heinze, G., Graf, S., Clavel, M.A., Khorsand, A., Bergler-Klein, J., et al. (2013) Two-Dimensional Strain for the Assessment of Left Ventricular Function in Low Flow-Low Gradient Aortic Stenosis, Relationship to Hemodynamics, and Outcome: A Substudy of the Multicenter TOPAS Study. Circulation: Cardiovascular Imaging, 6, 268-276. https://doi.org/10.1161/CIRCIMAGING.112.980201

[20] Kusunose, K., Goodman, A., Parikh, R., Barr, T., Agarwal, S., Popovic, Z.B., Grimm, R.A., Griffin, B.P. and Desai, M.Y. (2014) Incremental Prognostic Value of Left Ventricular Global Longitudinal Strain in Patients with Aortic Stenosis and Preserved Ejection Fraction. Circulation: Cardiovascular Imaging, 7, 938-945. https://doi.org/10.1161/CIRCIMAGING.114.002041

[21] Witkowski, T.G., Thomas, J.D., Debonnaire, P.J., Delgado, V., Hoke, U., Ewe, S.H., Versteegh, M.I., Holman, E.R., Schalij, M.J., Bax, J.J., Klautz, R.J. and Marsan, N.A. (2013) Global Longitudinal Strain Predicts Left Ventricular Dysfunction after Mitral Valve Repair. European Heart Journal-Cardiovascular Imaging, 14, 69-76. https://doi.org/10.1093/ehjci/jes155

[22] Maslow, A.D., Regan, M.M., Panzica, P., Heindel, S., Mashikian, J. and Comunale, M.E. (2002) Precardiopulmonary Bypass Right Ventricular Function Is Associated with Poor Outcome after Coronary Artery Bypass Grafting in Patients with Severe Left Ventricular Systolic Dysfunction. Anesthesia \& Analgesia, 95, 1507-1518. https://doi.org/10.1097/00000539-200212000-00009

[23] Haddad, F., Denault, A.Y., Couture, P., Cartier, R., Pellerin, M., Levesque, S., Lambert, J. and Tardif, J.C. (2007) Right Ventricular Myocardial Performance Index Predicts Perioperative Mortality or Circulatory Failure in High-Risk Valvular Surgery. Journal of the American Society of Echocardiography, 20, 1065-1072. https://doi.org/10.1016/j.echo.2007.02.017

[24] Ternacle, J., Gallet, R., Champagne, S., Teiger, E., Gellen, B., Dubois Rande, J.L., Gueret, P. and Lim, P. (2013) Changes in Three-Dimensional Speckle-TrackingDerived Myocardial Strain during Percutaneous Coronary Intervention. Journal of the American Society of Echocardiography, 26, 1444-1449. https://doi.org/10.1016/j.echo.2013.09.004

[25] Park, T.H., Nagueh, S.F., Khoury, D.S., Kopelen, H.A., Akrivakis, S., Nasser, K., Ren, G. and Frangogiannis, N.G. (2006) Impact of Myocardial Structure and Function Postinfarction on Diastolic Strain Measurements: Implications for Assessment of Myocardial Viability. American Journal of Physiology-Heart and Circulatory Physiology, 290, H724-H731. https://doi.org/10.1152/ajpheart.00714.2005

[26] Kalam, K., Otahal, P. and Marwick, T.H. (2014) Prognostic Implications of Global LV Dysfunction: A Systematic Review and Meta-Analysis of Global Longitudinal Strain and Ejection Fraction. Heart, 100, 1673-1680. https://doi.org/10.1136/heartjnl-2014-305538

[27] Lindqvist, P., Holmgren, A., Zhao, Y. and Henein, M.Y. (2012) Effect of Pericardial Repair after Aortic Valve Replacement on Septal and Right Ventricular Function. International Journal of Cardiology, 155, 388-393. https://doi.org/10.1016/j.ijcard.2010.10.049

[28] Maffessanti, F., Gripari, P., Tamborini, G., Muratori, M., Fusini, L., Alamanni, F., Zanobini, M., Fiorentini, C., Caiani, E.G. and Pepi, M. (2012) Evaluation of Right Ventricular Systolic Function after Mitral Valve Repair: A Two-Dimensional 
Doppler, Speckle-Tracking, and Three-Dimensional Echocardiographic Study. Journal of the American Society of Echocardiography, 25, 701-708. https://doi.org/10.1016/j.echo.2012.03.017

[29] Duncan, A.E., Sarwar, S., Kateby Kashy, B., Sonny, A., Sale, S., Alfirevic, A., Yang, D., Thomas, J.D., Gillinov, M. and Sessler, D.I. (2017) Early Left and Right Ventricular Response to Aortic Valve Replacement. Anesthesia \& Analgesia, 124, 406-418. https://doi.org/10.1213/ANE.0000000000001108

[30] Rost, C., Korder, S., Wasmeier, G., Wu, M., Klinghammer, L., Flachskampf, F.A., Daniel, W.G. and Voigt, J.U. (2010) Sequential Changes in Myocardial Function after Valve Replacement for Aortic Stenosis by Speckle Tracking Echocardiography. European Journal of Echocardiography, 11, 584-589. https://doi.org/10.1093/ejechocard/jeq017

[31] Badran, H.M., Soliman, M., Hassan, H., Abdelfatah, R., Saadan, H. and Yacoub, M. (2013) Right Ventricular Mechanics in Hypertrophic Cardiomyopathy Using Feature Tracking. Global Cardiology Science and Practice, 2013, 185-197. https://doi.org/10.5339/gcsp.2013.25

[32] Afonso, L., Briasoulis, A., Mahajan, N., Kondur, A., Siddiqui, F. and Siddiqui, S. (2015) Comparison of Right Ventricular Contractile Abnormalities in Hypertrophic Cardiomyopathy versus Hypertensive Heart Disease Using Two Dimensional Strain Imaging: A Cross-Sectional Study. The International Journal of Cardiovascular Imaging, 31, 1503-1509. https://doi.org/10.1007/s10554-015-0722-y

[33] Denault, A.Y., Couture, P., Beaulieu, Y., Haddad, F., Deschamps, A., Nozza, A., Page, P., Tardif, J.C. and Lambert, J. (2015) Right Ventricular Depression after Cardiopulmonary Bypass for Valvular Surgery. Journal of Cardiothoracic and Vascular Anesthesia, 29, 836-844. https://doi.org/10.1053/j.jvca.2015.01.011 